\title{
Minimal String Driven Cosmology and its Predictions
}

\author{
M.P.Infante ${ }^{(1),(2) *}$, N.Sánchez ${ }^{(1) \dagger}$ \\ (1) Observatoire de Paris-DEMIRM. 61, Avenue de l'Observatoire, 75014 Paris, FRANCE \\ (2) Dpto. Física Teórica, Univ. de Zaragoza. Pza.San Francisco, 50009 Zaragoza, SPAIN
}

\begin{abstract}
We present a minimal model for the Universe evolution fully extracted from effective String Theory. By linking this model with a minimal but well stablished observational information, we prove that it gives realistic predictions on early and current energy density and its results are compatible with General Relativity. Interestingly enough, the predicted current energy density is found $\Omega=1$ and in anycase with lower limit $\Omega \geq \frac{4}{9}$. On the other hand, the energy density at the exit of inflationary stage gives also $\left.\Omega\right|_{\text {inf }}=1$. This result shows an agreement with General Relativity (spatially flat metric gives critical energy density) within an unequivalent Non-Einstenian context (string low energy effective equations). The order of magnitude of the energy density-dilaton coupled term at the beginning of radiation dominated stage agrees with GUT scale. Without solving the known problems about higher order corrections and graceful exit of inflation, we find this model closer to the observational Universe properties than the current available string cosmology scenarii. At a more fundamental level, this model is by its construction close to the standard cosmological evolution, and it is driven selfconsistently by the evolution of the string equation of state itself. The inflationary String Driven stage is able to reach an enough amount of inflation, describing a Big Bang like evolution for the metric.
\end{abstract}

PACS Numbers: 98.80. Cq; 11.25.-w; 04.80.Cc; 98.80.-k

Report Numbers: hep-th/9910242; DFTUZ/99/15.

\footnotetext{
*E-mail: infante@mesunb.obspm.fr

${ }^{\dagger}$ E-mail: Norma.Sanchez@obspm.fr
} 


\section{Introduction}

The very early stages of the Universe must be described with physics beyond our current models. Around the Planck time, energy and sizes involved would require a quantum gravity treatment in order to account accurately for the physics at

such scale. String Theory appears as the most promising candidate for solving the first stages evolution. Until now, one does not dispose of a complete string theory, valid at the very beginning of the Universe neither the possibility of extracting so many phenomenological consequences from it. Otherwise, effective and selfconsistent string theories have been developed in the cosmological context in the last years ([1]-[5]). These approaches can be considered valid at the early stages inmediately after the Planck epoch and should be linked with the current stages, whose physics laws must be expected as the very low energy limits of the more complete laws in the early universe. Matters raise in this process. The Brans-Dicke frame, emerging naturally in the low energy effective string theories, includes both the General Relativity as well as the low energy effective string action as different particular cases. The former one takes place when the Brans-Dicke parameter $\omega_{B D}=\infty$ while the last one requires $\omega_{B D}=-1$ ([6]). Because of being extracted from different gravity theories, the effective string equations are not equivalent to the Einstein Equations. Since current observational data show agreement with General Relativity predictions, whatever another fundamental theory must recover it among their lowest energy limits, or at least must give results compatible with those extracted in Einstein frameworks. The great difficulties to incorporate string theory in a realistic cosmological framework are not so much expected at this level, but in the description of an early Universe evolution (string phase and inflation) compatible with the observational evolution information.

The scope of this paper is to present a minimal model for the Universe evolution completely extracted from selfconsistent string cosmology ([2]). In the following, we recall the selfconsistent effective treatments in string theory and the cosmological backgrounds arising from them. With these backgrounds, we construct a minimal model which can be linked with minimal observational Universe information. We analyse the properties of this model and confront it with General Relativity results. Although its simplicity, interesting conclusions are found about its capabilities as a predictive cosmological description. The predicted current energy density is found compatible with current observational results, since we have $\Omega \sim 1$ and in anycase $\Omega \geq \frac{4}{9}$. The energy density-dilaton coupled term at the beginnning of radiation dominated stage is found compatible with the order of magnitude typical of GUT scales $\rho e^{\phi} \sim 10^{90} \mathrm{erg}^{\mathrm{cm}}{ }^{-3}$. On the other hand, by defining the corresponding critical energy density, the energy density around the exit of inflation gives $\left.\Omega\right|_{\text {inf }}=1$. This result agrees with the General Relativity statement for which $k=0 \rightarrow \Omega=1$, but it is extracted in a Non-Einstenian context (the low energy string effective equations). No use of observational information neither further evolution Universe properties 
are needed in order to find this agreement, only the inflationary evolution law for the scale factor, dilaton and density energy.

Our String Driven Model is different from previously discussed scenarii in String Cosmology $([3])$. Until now, no complete description of the scale factor evolution from inmediately post-Planckian age until current time had been extracted in String Cosmology. The described inflationary stage, as here presented and interpretated, is also a new feature among the solutions given by effective string theory. The String Driven Model does not add new problems to the yet still open questions, but it provides a description closer and more naturally related to the observational Universe properties. The three stages of evolution, inflation, radiation dominated and matter dominated are completely driven by the evolution of the string equation of state itself. The results extracted are fully predictive without free parameters.

This paper is organized as follows: In Sections 2 and 3 we construct the Minimal String Driven Model. In Section 4 we discuss its main features: enough inflation, the evolution of the Hubble factor and the energy density predictions. We also discuss its main properties, differences and similarities with other string cosmology scenarii. In Section 5 we present our Conclusions.

\section{Minimal String Driven Model}

The String Driven Cosmological Background is a minimal model of the Universe evolution totally extracted from effective String Theory. We find the cosmological backgrounds from selfconsistent solutions of the effective string equations. Physical meaning of the model is preserved by linking it with a minimal but well stablished information about the evolution of the observational Universe. Two ways allowing extraction of cosmological backgrounds from string theory have been used. The first one is the low energy effective string equations plus the string action matter. Solutions of these equations are an inflationary inverse power evolution for the scale factor, as well as a radiation dominated behaviour. On the other hand, selfconsistent Einstein equations plus string matter, with a classical gas of strings as sources, give us again a radiation dominated behaviour and a matter dominated description. From both procedures, we obtain the evolution laws for an inflationary stage, a radiation dominated stage and a matter dominated stage. These behaviours are asymptotic regimes not including strictly the transitions among stages. By modelizing the transitions in an enoughly continuous way, we construct a step-by-step minimal model of evolution. In this whole and next sections, unless opposite indication, the metric is defined in lenght units. Thus, the $(0,0)$ component is always time coordinate $\mathcal{T}$ multiplied by constant $c, t=c \mathcal{T}$. Derivatives are taken with respect to this coordinate $t$. 


\subsection{The Low Energy Effective String Equations}

We work with the low energy effective string action (that means, to the lowest order in expansion of powers of $\alpha^{\prime}$ ), which in the Brans-Dicke or string frame can be written as $(\llbracket 1],[2],[3])$ :

$$
S=-\frac{c^{3}}{16 \pi G_{D}} \int d^{d+1} x \sqrt{|g|} e^{-\phi}\left(R+\partial_{\mu} \phi \partial^{\mu} \phi-\frac{H^{2}}{12}+V\right)+S_{M}
$$

where $S_{M}$ is the corresponding action for the matter sources, $H=d B$ is the antisymmetric tensor field strength and $V$ is a constant vanishing for some critical dimension. The dilaton field $\phi$ depends explicitly only upon time coordinate and its potential will be considered vanishing. $D$ is the total spacetime dimension. We consider a spatially flat background and we write the metric in synchronous frame $\left(g_{00}=1, g_{0 i}=0=g_{0 a}\right)$ as:

$$
g_{\mu \nu}=\operatorname{diag}\left(1,-a^{2}(t) \delta_{i j}\right)
$$

here $\mu, \nu=0 \ldots(D-1)$ and $i, j=1 \ldots(D-1)$. The string matter is included as a classical source which stress energy tensor in the perfect fluid approximation takes the form:

$$
T_{\mu}{ }^{\nu}=\operatorname{diag}\left(\rho(t),-P(t) \delta_{i}{ }^{j}\right)
$$

where $\rho$ and $P$ are the energy density and pressure for the matter sources respectively.

The low energy effective string equations are obtained by extremizing the variation of the effective action $S$ (豆 with respect to the metric $g_{\mu \nu}$, the dilaton field $\phi$ and the antisymmetric field $H_{\mu \nu \alpha}$ and taking into account the metric (2) and matter sources (3). We will consider that the antisymmetric tensor $H_{\mu \alpha \beta}$ as well as the potential $B$ vanish. By defining $H=\frac{\dot{a}}{a}$ and the shifted expressions for the dilaton

$\bar{\phi}=\phi-\ln \sqrt{|g|}$, matter energy density $\bar{\rho}=\rho a^{d}$ and pressure $\bar{p}=P a^{d}$, we obtain the low energy effective equations L.E.E. ([2], [3]):

$$
\begin{aligned}
& \dot{\bar{\phi}}^{2}-2 \ddot{\bar{\phi}}+d H^{2}=0 \\
& \dot{\bar{\phi}}^{2}-d H^{2}=\frac{16 \pi G_{D}}{c^{4}} \bar{\rho} e^{\bar{\phi}} \\
& 2(\dot{H}-H \dot{\bar{\phi}})=\frac{16 \pi G_{D}}{c^{4}} \bar{p} e^{\bar{\phi}}
\end{aligned}
$$

The shifted expressions have the property to be invariants under the transformations related to the scale factor duality simmetry $\left(a \rightarrow a^{-1}\right)$ and time reflection $(t \rightarrow-t)$. Following this, if $(a, \phi)$ is a solution of the effective equations, the dual expression $(\hat{a}, \hat{\phi})$ obtained as:

$$
\hat{a}_{i}=a_{i}{ }^{-1} \quad, \quad \hat{\phi}=\phi-2 \ln a_{i}
$$

is also a solution of the same system of equations. 


\subsubsection{String Driven Inflationary Stage Solution}

The inflationary stage appears as a new selfconsistent solution of the low energy effective equations (4) sustained by a gas of stretched or unstable string sources as developed in [2] and also in [3]. This kind of string behaviour is characterized by a negative pressure and positive energy density, both growing in absolute value with the scale factor $([2],[4],[8],[9])$. Strings in curved backgrounds satisfy the perfect fluid equation of state $P=(\gamma-1) \rho$ where $\gamma$ is different for each one of the generic three different string behaviours in curved spacetimes ([2]). For the unstable (stretched) string behaviour, it holds $\gamma_{u}=\frac{D-2}{D-1}(\mathbb{4} \mid$ ). Thus, the equation of state for these string sources in the metric (2) is given by ([2]):

$$
P=-\frac{1}{d} \rho
$$

We find the following selfconsistent solution for the set of effective equations (4) with the matter sources eq.(6):

$$
\begin{aligned}
a(t) & =A_{I}\left(t_{I}-t\right)^{-Q} \quad 0<t<t_{b}<t_{I} \quad Q=\frac{2}{d+1} \\
\phi(t) & =\phi_{I}+2 d \ln a(t) \\
\rho(t) & =\rho_{I}(a(t))^{(1-d)} \\
P(t) & =-\frac{1}{d} \rho(t)=-\frac{\rho_{I}}{d}(a(t))^{(1-d)}
\end{aligned}
$$

Notice that here $t$ is the cosmic time coordinate, running on positive values such that the parameter $t_{I}$ is greater than the end of the string driven inflationary regime at time $t_{b}, d$ is the number of expanding spatial dimensions; $\rho_{I}, \phi_{I}$ are integration constants and $A_{I}, t_{I}$ parameters to be fixed by the further evolution of scale factor.

Although the time dependence obeys a power function, this String Driven solution is an inflationary inverse power law proper to string cosmology. This solution describes an inflationary stage with accelerated expansion of scale factor since $H>0, \dot{H}>0$ and can be considered superinflationary, since $\ddot{a}(t)$ increases with time. However, notice the negative power of time and the decreasing character of the interval $\left(t_{I}-t\right)$. Notice also that the string energy density $\rho(t)$ and the pressure $P(t)$ have a decreasing behaviour when the scale factor grows.

\subsubsection{String Driven Radiation Dominated Stage Solution}

This stage is obtained by following the same procedure above described, but by considering now a gas of strings with dual to unstable behaviour. Dual strings 
propagate in curved spacetimes obeying a typical radiation type equation of state ( [2], [3]).

$$
P=\frac{1}{d} \rho
$$

This string behaviour and the dilaton "frozen" at constant value ( $\phi=$ constant) gives us the scale factor for the radiation dominated stage:

$$
\begin{aligned}
a(t) & =A_{I I} t^{R} \quad R=\frac{2}{d+1} \\
\phi(t) & =\phi_{I I} \\
\rho(t) & =\rho_{I I}(a(t))^{-(1+d)} \\
P(t) & =\frac{1}{d} \rho(t)=\frac{\rho_{I I}}{d}(a(t))^{-(1+d)}
\end{aligned}
$$

here $\phi_{I I}, \rho_{I I}$ are integration constants, and $A_{I I}$ a parameter to be fixed by the evolution of the scale factor.

\subsection{Selfsustained String Universes in General Relativity}

As shown in ref.[2], [1 and [5], string solutions in curved spacetimes are selfconsistent solutions of General Relativity equations, in particular in a spatially flat, homogeneus and isotropic background:

$$
d s^{2}=d t^{2}-a(t)^{2} d x^{2}
$$

where the Einstein equations take the form:

$$
\frac{1}{2} d(d-1) H^{2}=\rho \quad, \quad(d-1) \dot{H}+P+\rho=0
$$

As before, the matter source is described by a gas of non interacting classical strings (neglecting splitting and coalescing interactions). This gas obeys an equation of state including the three different possible behaviours of strings in curved spacetimes: unstable, dual to unstable and stable. Let be $\mathcal{U}, \mathcal{D}$ and $\mathcal{S}$ the densities for strings with unstable, dual to unstable and stable behaviours respectively. Taking into account the properties of each behaviour ([2]), the density energy and the pressure of the string gas are described by:

$$
\rho=\frac{1}{(a(t))^{d}}\left(\mathcal{U} a(t)+\frac{\mathcal{D}}{a(t)}+\mathcal{S}\right) \quad, \quad P=\frac{1}{d} \frac{1}{(a(t))^{d}}\left(\frac{\mathcal{D}}{a(t)}-\mathcal{U} a(t)\right)
$$

Equations (12) are qualitatively correct for every $t$ and become exact in the asymptotic cases, leading to obtain the radiation dominated behaviour of the scale 
factor, as well as the matter dominated behaviour. In the limit $a(t) \rightarrow 0$ and $t \rightarrow 0$, the dual to unstable behaviour dominates in the equations (12) and gives us:

$$
\rho(t) \sim \mathcal{D}(a(t))^{-(d+1)} \quad, \quad P(t) \sim \frac{1}{d} \mathcal{D}(a(t))^{-(d+1)}
$$

This behaviour is characterized by positive string density energy and pressure, both growing when the scale factor approaches to 0. Dual to unstable strings behave in similar way to massless particles, i.e. radiation. Solving selfconsistently the Einstein equations (11) with sources following eqs.(13), the scale factor solution takes the form:

$$
a(t) \sim\left(\frac{2 \mathcal{D}}{d(d-1)}\right)^{\frac{1}{d+1}}\left(\frac{d+1}{2}\right)^{\frac{2}{d+1}}\left(t-t_{I I}\right)^{R} \quad, \quad R=\frac{2}{d+1}
$$

this describes the evolution of a Friedmann-Robertson-Walker radiation dominated Universe, the time parameter $t_{I I}$ will be fixed by further evolution of the scale factor. On the other hand, studying the opposite limit $a(t) \rightarrow \infty, t \rightarrow \infty$ and taking into account the behaviour of the unstable density $\mathcal{U}$ which vanishes for $a(t) \rightarrow \infty$ [2], the stable behaviour becomes dominant and the equation of state reduces to:

$$
\rho \sim \mathcal{S}(a(t))^{-d} \quad, \quad P=0
$$

The stable behaviour gives a constant value for the string energy, that is, the energy density evolves as the inverse volume decreasing with growing scale factor, while the pressure vanishes. Thus, stable strings behave as cold matter. Again, from solving eqs.(11) with eqs.(15), the solution of a matter dominated stage emerges:

$$
a(t) \sim\left(\frac{d}{(d-1)} \frac{\mathcal{S}}{2}\right)^{\frac{1}{d}}\left(t-t_{I I I}\right)^{M} \quad, \quad M=\frac{2}{d}
$$

We construct in the next sections a model with an inflationary stage described by the String Driven solution (see eq.(7)), followed by a radiation dominated stage (see eq.(9)) and a matter dominated stage (see eq.(16)). We will consider the dilaton field remain practically constant and vanishing from the exit of inflation until the current time, as suggested in the String Driven Radiation Dominated Solution. It must be noticed that the same solution for the radiation dominated stage emerges from the treatment with dilaton field and without it (general relativity plus string equation of state), allowing us to describe qualitatively the evolution of the universe by means of these scale factor asymptotic behaviours.

\section{Scale Factor Transitions}

Taking the simplest option, we consider the "real" scale factor evolution mini- 
mally described as:

$$
\begin{array}{rlr}
a_{I}(t) & =A_{I}\left(t_{I}-t\right)^{-Q} \quad t \in\left(t_{i}, t_{r}\right) \\
a_{I I}(t) & =A_{I I} t^{R} \quad t \in\left(t_{r}, t_{m}\right) \\
a_{I I I}(t) & =A_{I I I} t^{M} \quad t \in\left(t_{m}, t_{0}\right)
\end{array}
$$

with transitions at least not excessively long at the beginning of radiation dominated stage $t_{r}$ and of matter dominated stage $t_{m}$. We also define a beginning of inflation at $t_{i}$, and $t_{0}$ is the current time. It would be reasonable do not have instantaneous and continuous transitions at $t_{r}$ and $t_{m}$ for the stages extracted in the above section, since the detail of such transitions is not provided by the effective treatments here used. One can suspects the existence of very brief intermediate stages at least at the end of the inflationary stage $\left(t \in\left(t_{b}, t_{r}\right)\right)$, as we will discuss in the next section, and also at the end of radiation dominated stage $\left(t \sim t_{m}\right)$. The dynamics of these transitions is unknown and not easy to modelize, it introduces in anycase free parameters ([[]). In order to construct an evolution model for the scale factor, it is compatible with the current level of knowledge of the theory to suppose the transitions very brief. We will merge our lack of knowledge on the real transitions by means of descriptive temporal variables ([7]) in function of which the modelized transitions at $\overline{t_{1}}$ and $\overline{t_{2}}$ are instantaneous and continuous. We link this descriptive scale factor with the minimal information about the evolution of the observational Universe; we consider the standard values for cosmological times: the radiation-matter transition held about $\mathcal{T}_{m} \sim 10^{12} \mathrm{~s}$, the beginning of radiation stage at $\mathcal{T}_{r} \sim 10^{-32}$ s and the current age of the Universe $\mathcal{T}_{0} \sim H_{0}{ }^{-1} \sim 10^{17} \mathrm{~s}$ (The exact numerical value of $\mathcal{T}_{0}$ turns out not crucial here). We impose also to our description satisfy the same scale factor expansion (or scale factor ratii) reached in each one of the three stages considered in the real model (1). It is also convenient to fix the temporal variable of the third (and current) stage $t$ with our physical time (multiplied by $c$ ). Explicit computations can be found in [7] and led finally to the following scale factor in cosmic time-type variables:

$$
\begin{aligned}
\overline{a_{I}}(\overline{\bar{t}}) & =\overline{\bar{A}_{I}}\left(\overline{\overline{t_{I}}}-\overline{\bar{t}}\right)^{-Q} & \overline{\bar{t}_{i}}<\overline{\bar{t}}<\overline{t_{1}} \\
\overline{a_{I I}}(\bar{t}) & =\overline{A_{I I}\left(\bar{t}-\bar{t}_{I I}\right)^{R}} & \overline{t_{1}}<\bar{t}<\overline{t_{2}} \\
a_{I I I}(t) & =A_{I I I}(t)^{M} & \overline{t_{2}}<t<t_{0}
\end{aligned}
$$

with continuous transitions at $\overline{t_{1}}$ and $\overline{t_{2}}$ both the scale factor and first derivatives with respect to the variables $\overline{\bar{t}}, \bar{t}$ and $t$. The parameters $\overline{\bar{t}}_{I}, \overline{\bar{A}}_{I}, \bar{t}_{I I}, A_{I I I}$ can be written in function of $\overline{A_{I I}}$ and transition times using the matching conditions. In terms of standard observational values, the transitions $\overline{t_{1}}, \overline{t_{2}}$ and the beginning of the inflationary stage description $\overline{\bar{t}}_{i}$ are expressed as:

$$
\begin{aligned}
& \overline{t_{1}}=\frac{R}{M} t_{r}+\left(1-\frac{R}{M}\right) t_{m} \quad, \quad \overline{t_{2}}=t_{m} \\
& \overline{\overline{t_{i}}}=\left(\frac{R}{M}-\frac{Q}{M} \frac{t_{r}-t_{i}}{t_{I}-t_{r}}\right) t_{r}+\left(1-\frac{R}{M}\right) t_{m}
\end{aligned}
$$


The parameters of the scale factor (2) can be written also in terms of the observational values $t_{r}, t_{m}$ and the global scale factor $\overline{A_{I I}}$ :

$$
\begin{aligned}
& \overline{\overline{t_{I}}}=t_{r}\left(\frac{R}{M}+\frac{Q}{M}\right)+t_{m}\left(1-\frac{R}{M}\right) \quad, \quad t_{I I}^{-}=\left(1-\frac{R}{M}\right) t_{m} \\
& \overline{\bar{A}}_{I}=\overline{A_{I I}}\left(\frac{Q}{M}\right)^{Q}\left(\frac{R}{M}\right)^{R} t_{r}{ }^{R+Q} \quad, \quad A_{I I I}=\overline{A_{I I}}\left(\frac{R}{M}\right)^{R} t_{m}{ }^{R-M}
\end{aligned}
$$

The time variable $\overline{\bar{t}}$ of inflationary stage and $\bar{t}$ of radiation stage are not a priori exactly equal to the physical time coordinate at rest frame (multiplied by $c$ ), but transformations (dilatation plus translation) of it. The low energy effective action equations from where the scale factor, dilaton and energy density have been extracted, allow these transformations. With this treatment of the cosmological scale factor, we will attain computations free of "by hand" added parameters and with full predictability as can be seen in the next sections.

The last point is to make an approach for the dilaton field. This is considered practically constant from the beginning of the radiation dominated stage until the current time. The dilaton field increases during the inflationary string driven stage. Its value can be supposed coincident with the value at exit inflation time in a sudden but not continuous transition, since its temporal derivatives can not match this asymptotic behaviour. $\phi_{I I}=\phi\left(t_{r}\right) \equiv \phi_{1}$. Remember the expression for the dilaton in inflation dominated stage,

$$
\phi_{I I}=\phi_{I}+2 d \ln a\left(t_{r}\right)
$$

The scale factor can be written also in terms of the conformal time variable

$d \eta=\frac{d t}{a(t)}$ defined for each stage. Detailed computation can be found elsewhere ( [7], [10]) and are not needed here.

\section{Properties of the String Driven Model}

\subsection{Enough Inflation}

The loose ends in standard FRW cosmology are the flatness problem or why the current spatial curvature $k$ is so little and the corresponding $\Omega$ so close to 1 ; the horizon problem and causality related considerations and the large scale homogeneity of Universe and related perturbation considerations. The inflationary paradigm with an exponential expansion period answers these questions provided the inflationary expansion reaches an enough number of $e$-folders, usually considered 
among $80-100$. Any other cosmological theory predicting an inflationary stage should have to answer these questions. As the minimum requirement in this sense, a compatibility with enough amount of inflation must be required. We discuss now if enough inflation could be provided by the String Driven inflationary stage. In the comoving coordinates, enough inflation can be simply produced if the additive parameter $t_{I}$ is found sufficiently near and above the inflation-radiation dominated transition time $t_{r}$. In this way, by increasing the time, the scale factor inflates by approaching a pole type singularity. But the singularity is not reached neither passed throw, because the exit of inflation stage happens before.

Notice also that both the scale factor and dilaton increase with time, as well as the spacetime curvature since it is proportional to $H$. As a consequence, the dynamics of the stage could lead to break the conditions giving rise to the low energy effective regime. Let be $t_{b}$ the instant when this breaking in the low energy effective regime happens and, as consequence, the string driven inflationary evolution ends. What kind of evolution follows is an open question, a more complete treatment would be neccesary in order to describe the scale factor in a higher order regime, where string effects would play a strong role. By consistency with the time scales, it is reasonable to suppose a very short interval between the end of the string driven inflation and the beginning of the standard radiation dominated cosmology. One can suspects the existence of a very brief intermediate stage at the end of the inflationary stage at nearly constant curvature $\left(t \in\left(t_{b}, t_{r}\right)\right)$. Due to its briefness and supposed bounded curvature controlled by higher-order string corrections, not so great expansion would appear in the stage not governed by the L.E.E..

Let us impose the String Driven inflationary stage to reach an arbitrary number $f$ of e-folds between the beginning at $t_{i}$ and the comeback to L.E.E. at $t_{r}$ :

$$
\frac{a_{I}\left(t_{r}\right)}{a_{I}\left(t_{i}\right)} \sim e^{f}
$$

This condition gives us the relation:

$$
1+\frac{t_{r}-t_{i}}{t_{I}-t_{r}} \sim e^{\frac{f}{Q}}
$$

By defining the parameter $\epsilon$ related with the value $t_{I}$ through $t_{I}=t_{r}(1+\epsilon)$ and $\delta$ relating the inflation duration and its exit, the enough inflation condition eq.(1) translates as:

$$
1+\frac{\delta}{\epsilon} \sim e^{\frac{f}{Q}}
$$

that, in good approximation, gives us $\epsilon \sim \delta e^{-\frac{f}{Q}}$.

The dilaton field increases during the inflationary stage, however its effect in breaking the L.E.E. regime is not given by the exit inflation conditions but by the 
beginning inflation ones. We have modelized a time $t_{i}$ being the beginning of an inflationary stage close to Planck time $t_{P} \sim 10^{-43} \mathrm{~s}$. In this inflationary stage, from eqs.(7) the dilaton field is $\phi(t)=\phi_{I}+2 d \ln a(t)$. Therefore, the dilaton increases during the inflationary stage between $t_{i}$ and $t_{r}$ a ratio:

$$
\frac{\phi\left(t_{r}\right)}{\phi\left(t_{i}\right)}=\frac{\phi_{I}+2 d \ln a\left(t_{r}\right)}{\phi_{I}+2 d \ln a\left(t_{i}\right)}
$$

Thus, if an enough amount of inflation tooks place, for instance $a\left(t_{r}\right)=e^{f} a\left(t_{i}\right)$, the ratio in the dilaton field is given by the expression:

$$
\frac{\phi\left(t_{r}\right)}{\phi\left(t_{i}\right)}=1+\frac{f}{\log _{10} e} \frac{2 d}{\left(\phi_{I}+2 d \ln a\left(t_{i}\right)\right)}
$$

where we notice the lineal dependence with the number of e-folds $f$ and the inverse dependence of scale factor at the beginning of inflation stage $a\left(t_{i}\right)$.

No need to place this singularity on the big bang (around $t \rightarrow 0$ ) is found from the effective equations. Still more: the beginning $t_{i}$ of the String Driven inflationary stage is practically irrelevant. The usual inflationary paradigm considers between 60100 e-folds enough to solve the problem of large scale homogeneity. This quantity is true for standard (exponential De Sitter) inflation, but we can consider it enoughly restrictive. In anycase, we realize that String Driven inflation can, in principle, provide whatever amount of inflation, since a finite value of $f$ gives a finite relation among $t_{I}$ and $t_{r}$ too. One would expect the value of $t_{I}$ given from theory and predicting the right amount of inflation. In our limited minimal string driven model, we prove the compatibility of our inflationary description with the usual standard inflation values.

In the figure [1] we show the scale factor evolution for a De $\operatorname{Sitter} \operatorname{stage}(D S)$, an usual power law inflationary stage $(P L)$ and the String Driven inflationary stage $(S D)$.

We consider now the duration of inflation. In the descriptive coordinate $\overline{\bar{t}}$, it means $\left.\Delta \overline{\bar{t}}\right|_{i n f}=\bar{t}_{1}-\overline{\bar{t}}_{i}$. From eqs.(3) is seen:

$$
\left.\Delta \overline{\bar{t}}\right|_{i n f}=\left.\frac{Q}{M} \frac{t_{r}}{t_{I}-t_{r}} \Delta t\right|_{i n f}
$$

where $\left.\Delta t\right|_{\text {inf }}=t_{r}-t_{i}$ and remember $Q$ and $M$ are positive values and $t_{I}>t_{r}$. Coherently, we obtain a positive value for the duration of inflation in descriptive variables. Notice that as long as $t_{I} \rightarrow t_{r}$, this duration experiments a greater dilatation. In fact, if we have in general $t_{I}=t_{r}(1+\epsilon)$, then:

$$
\left.\Delta \overline{\bar{t}}\right|_{i n f}=\left.\frac{Q}{M} \frac{1}{\epsilon} \Delta t\right|_{i n f}
$$




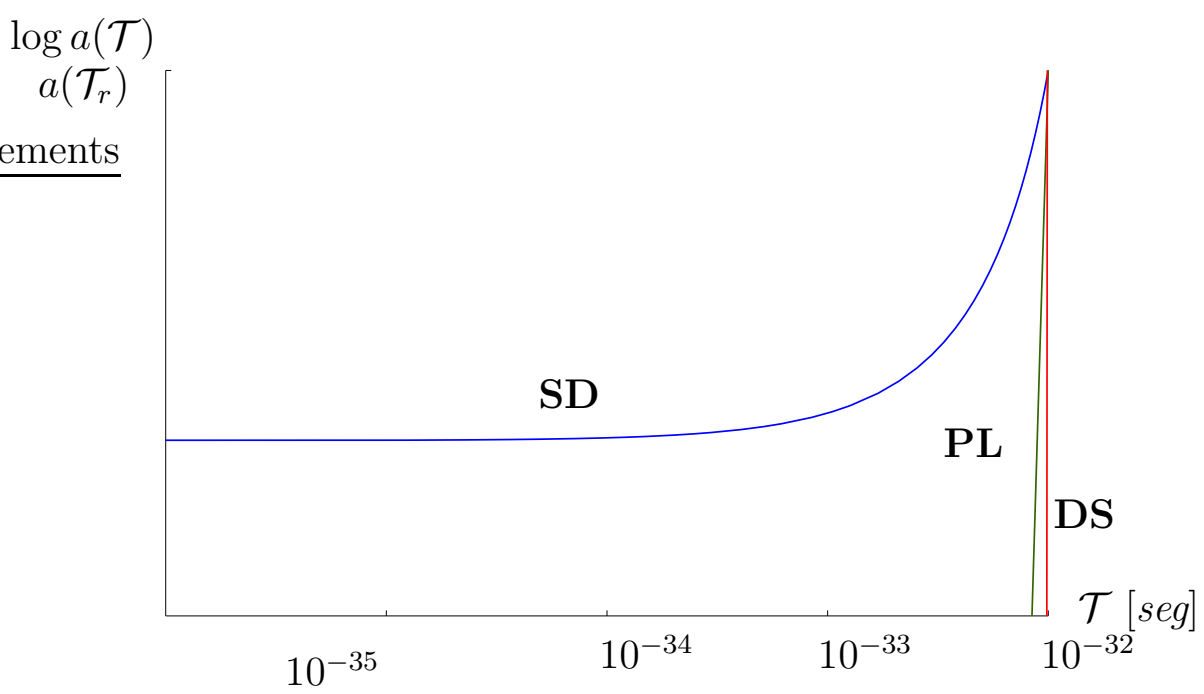

Figure 1: Comparison among different inflationary evolution laws

Representation of the inflation amount reached by a De Sitter $(D S)$, an usual Power Law $(P L)$ and a String Driven $(S D)$ inflationary stages. The three scale factors have been normalized at $\mathcal{T}_{r}$. For enough inflation $\left(t_{I} \rightarrow t_{r}\right)$, the $(S D)$ behaviour becomes very much sharper.

Since enough inflation requires $\epsilon<<1$, the duration in descriptive variables increases so much in order to reproduce the expansive behaviour of the scale factor. For instance, for an expansion of order $\sim e^{f}$, the last expression becames:

$$
\left.\Delta \overline{\bar{t}}\right|_{i n f}=\left.\frac{Q}{M} e^{\frac{f}{Q}} \Delta t\right|_{i n f}
$$

Such enormous dilatation in the descriptive variables is reached by means of transformation of the transition times. From eqs.(3) we see that $\bar{t}_{1} \sim t_{m}$ and always $\bar{t}_{1}>0$, meanwhile $\overline{\bar{t}}_{i}$ can decrease to large negative values, enlarging in this way the duration of the stage in the descriptive variables. By using $t_{I}=t_{r}(1+\epsilon)$ and $t_{r}-t_{i}=\delta t_{r}$, the condition $\overline{\bar{t}}_{i}<0$ holds when:

$$
\frac{\delta}{\epsilon} \geq \frac{1}{Q}\left(R+(M-R) \frac{t_{m}}{t_{r}}\right)
$$

With the standard transition times given in sections above, the r.h.s. of eq.(3i) is $\sim 10^{43}$. In the l.h.s. we find $\delta \rightarrow 1$ and $\epsilon \rightarrow 0$. For beginning of inflation around the Planck time $\left(t_{i} \rightarrow t_{P}\right)$ and enough inflation $(f \sim 80)$, we have $\frac{\delta}{\epsilon} \sim 10^{87}$ and equation (3) is satisfied. This feature is needed in order to attain the required amount of inflation in the descriptive variables, because it requires a great dilatation of the inflation duration $\left.\left.\Delta \overline{\bar{t}}\right|_{\text {inf }} \sim 10^{87} \Delta t\right|_{\text {inf }}$. The temporal descriptive coordinate $\overline{\bar{t}}$ runs on both positive and negative values, but the proper cosmic time runs always on positive values. 
The matter dominated stage description presents the same duration that the real one since it runs on proper cosmic time. The duration of the radiation dominated stage in descriptive variables suffers a slight contraction with respect to duration in proper time $\left.\Delta \bar{t}\right|_{\text {rad }}=\left.\frac{R}{M} \Delta t\right|_{\text {rad }}$. For our model in three spatial dimensions, this value is $\frac{R}{M}=\frac{1}{3}$

\subsection{Evolution of the Hubble Factor}

The Hubble factor in the model constructed with the String Driven inflationary stage has the following behaviours in each stage:

$$
\begin{aligned}
\left.H(t)\right|_{\text {inf }} & =c Q\left(t_{I}-t\right)^{-1} \\
\left.H(t)\right|_{\text {rad }} & =c R\left(t-t_{I I}\right)^{-1} \\
\left.H(t)\right|_{\text {mat }} & =c M(t)^{-1}
\end{aligned}
$$

In the figure [2] we can observe the evolution of Hubble factor in the minimal String Driven cosmological model. Notice the growth of $H(t)$ around the exit of inflation. Since the Hubble factor is proportional to spacetime curvature, the region around $t_{r}$ is a highly curved regime, where the L.E.E. regime should transitorily break down. Notice also the nearly constant curvature at the beginning of the inflation stage

\subsection{Energy Density Predictions}

As can be easily seen, from eq.(田) we have:

$$
\dot{\bar{\phi}}^{2}-2 \ddot{\bar{\phi}}+d H^{2}=0 \quad, \quad \dot{\bar{\phi}}^{2}-d H^{2}=\frac{16 \pi G_{D}}{c^{4}} \bar{\rho} e^{\bar{\phi}}
$$

Both equations give:

$$
\ddot{\bar{\phi}}-d H^{2}=\frac{8 \pi G_{D}}{c^{4}} \bar{\rho} e^{\bar{\phi}}
$$

By substituting the shifted expressions for the dilaton $\bar{\phi}=\phi-\ln \sqrt{|g|}$ and matter energy density $\bar{\rho}=\rho a^{d}$, the above equation yields:

$$
\ddot{\phi}-d\left(\dot{H}+H^{2}\right)=\frac{8 \pi G_{D}}{c^{4}} \rho e^{\phi}
$$

Eq.(5) can be considered as the generalization of the Einstein equation in the framework of low energy effective string action. This equation will allow us extract some predictions on the energy density evolution in our minimal model. 


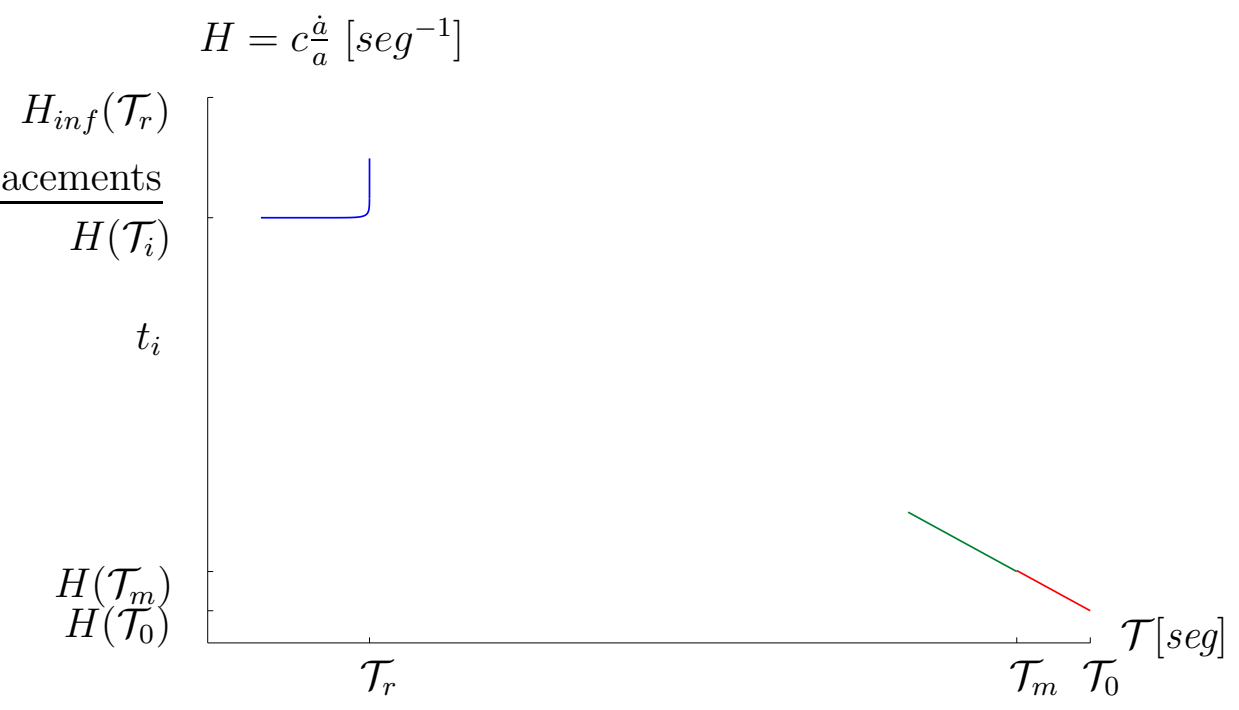

Figure 2: Representation of Hubble factor in String Driven Cosmology

Representation of the Hubble factor evolution in the minimal String Driven cosmological model. During the inflationary stage, the Hubble factor remains almost constant until the neighbourhood of the transition to radiation dominated stage. Just before it, the Hubble factor has an explosive but bounded growth. During the radiation dominated and matter dominated stages, the Hubble factor decreases with a nearly constant slope.

\subsubsection{Energy Density at the Exit of Inflation}

By introducing the String Driven Solution for $a(t), \phi(t), \rho(t)$ (eqs.(77)) in eq.(55), we obtain a relation for the integration constants $\rho_{I}$ and $\phi_{I}$ :

$$
\rho_{I} e^{\phi_{I}}=\frac{c^{4}}{8 \pi G_{D}} \frac{2 d(d-1)}{(d+1)^{2}} A_{I}^{-(1+d)}
$$

Now, is easy to find the relation between $\rho_{I}, e^{\phi_{I}}$ and the values of the energy density $\rho_{1}$ and dilaton field $\phi_{1}$ at the end of inflation stage. We can compute these values either with the real scale factor $a_{I}(t)$ or with the description $\overline{\overline{a_{I}}}(\overline{\bar{t}})$. By defining $\rho_{1}=\rho\left(a_{I}\left(t_{r}\right)\right)=\rho\left(\overline{\bar{a}_{I}}\left(\overline{t_{1}}\right)\right)$ and $\phi_{1}=\phi\left(a_{I}\left(t_{r}\right)\right)=\phi\left(\overline{\bar{a}_{I}}\left(\overline{t_{1}}\right)\right)$ and making use of eqs.(可), we can write:

$$
\rho_{1} e^{\phi_{1}}=\rho_{I} e^{\phi_{I}} A_{I}{ }^{1+d}\left(t_{I}-t_{r}\right)^{-Q(1+d)}
$$

Now, with the information about the evolution of the scale factor and the descriptions in each stage, it is possible to relate this expression with observational cosmological parameters. In fact, we must understand eq.(7) as one obtained in the description of inflationary stage:

$$
t_{I}-t_{r} \rightarrow \overline{\overline{t_{I}}}-\overline{t_{1}}=\frac{Q}{M} t_{r}
$$


For the String Driven solution, the exponents have the values $Q=\frac{2}{d+1}, R=\frac{2}{d+1}$, $M=\frac{2}{d}$. With these expressions we obtain the density-dilaton coupling at the end of inflation:

$$
\rho_{1} e^{\phi_{1}}=\frac{c^{4}}{8 \pi G_{D}} \frac{2(d-1)}{d} t_{r}^{-2}
$$

With $t_{r}=c \mathcal{T}_{r}$ where $\mathcal{T}_{r} \sim 10^{-32} s$, this expression gives the numerical value:

$$
\rho_{1} e^{\phi_{1}}=7.110^{90} \mathrm{erg} \mathrm{cm}^{-3}
$$

It must be noticed that the same result can be achieved from the Radiation Dominated Stage, due to the continuity of the scale factor, of the density energy and the dilaton field at the transition time $t_{r}$.

It must be noticed also an interesting property of this energy density-dilaton coupled term. We can extend the General Relativity treatment and define $\left.\Omega\right|_{\text {inf }}$ as this coupled quantity in critical energy density units, where the critical energy density $\rho_{c}(t)$ for our spatially flat metric is $\rho_{c}(t)=\frac{3 c^{2}}{8 \pi G} H(t)^{2}$. We compute the corresponding $\rho_{c}$ at the moment of the exit of inflation:

$$
\left.\rho_{c}\right|_{\overline{t_{1}}}=\frac{3 c^{2}}{8 \pi G} H\left(\overline{t_{1}}\right)=\frac{3 c^{4}}{8 \pi G} M^{2} t_{r}{ }^{-2}
$$

With this, it is easily seen:

$$
\left.\Omega\right|_{i n f}=\frac{\rho_{1} e^{\phi_{1}}}{\left.\rho_{c}\right|_{\overline{t_{1}}}}=\frac{2}{3} \frac{d-1}{d} \frac{1}{M^{2}}
$$

where $M$ is given by eq.(16). It gives for our model in the three dimensional case $\left.\Omega\right|_{\text {inf }}=1$.

Here we have used the descriptive variables in order to link the scale factor transitions with observational transition times. But the conclusion $\left.\Omega\right|_{\text {inf }}=1$ is independent from this choice. In fact, we have computed it again in the proper cosmic time of the inflationary stage and taking the corresponding values at the beginning of the radiation dominated stage $t_{r}$. From eq.(7) and $H(t)=Q\left(t_{I}-t\right)^{-1}$, we have:

$$
\left.\Omega\right|_{i n f}=\frac{1}{3 Q}\left(\frac{2}{Q}-1-d Q\right)
$$

$Q$ is given by the String Driven solution eq.(7) and with it, we recover $\left.\Omega\right|_{\text {inf }}=1$. In fact, the same result could be achieved also by evaluating $\left.\Omega\right|_{\text {inf }}$ exactly at the end of String Driven inflationary stage, whatever this time may be. That means, we have a prediction non-dependent of whenever the exit of inflation happens.

In the proper cosmic time coordinates, $\left(t_{I}-t_{r}\right)$ is a very little value. But independently from this, the coupled term energy density-dilaton at the exit of 
inflation gives the value one in the corresponding critical energy density units. The value of the critical energy density is computed following the Einstein Equations for spatially flat metrics, but solely the low energy effective string equations give the expression for the coupled term eq.(5) and the String Driven solution itself. In this last point, no use of further evolution, linking with observational results neither standard cosmology have been made. This enable us to affirm that in the L.E.E. treatment, the relation between spatial curvature and energy density holds as in General Relativity, at least for the spatially flat case $(k=0 \rightarrow \Omega=1)$. This result means to recover a General Relativity prescription within a Non-Einstenian framework.

\subsubsection{Predicted Current Values of Energy Density and Omega.}

Thus, we can obtain the corresponding current value of $\rho_{0} e^{\phi_{0}}$ in units of critical energy density or contribution to $\Omega$.

To proceed, we remember that the evolution of the density energy in the matter dominated stage follows $\rho \sim a(t)^{-3} \sim t^{-2}$ then, at the beginning of matter dominated stage we would have

$$
\rho_{m}=\left(\frac{\mathcal{T}_{m}}{\mathcal{T}_{0}}\right)^{-2} \rho_{0}
$$

On the other hand, in the radiation dominated stage, the density behaviour is: $\rho \sim a(t)^{-4} \sim t^{-2}$ which gives for the energy density

$$
\rho_{1}=\left(\frac{\mathcal{T}_{r}}{\mathcal{T}_{m}}\right)^{-2} \rho_{m}
$$

That is,

$$
\rho_{0}=\left(\frac{\mathcal{T}_{r}}{\mathcal{T}_{0}}\right)^{2} \rho_{1}
$$

Considering that the dilaton field has been remained almost constant since the end of inflation $\phi_{0} \sim \phi_{r}$, we have

$$
\Omega=\frac{\rho_{0} e^{\phi_{0}}}{\rho_{c}}=\left(\frac{\mathcal{T}_{r}}{\mathcal{T}_{0}}\right) e^{\phi_{1}} \frac{\rho_{1}}{\rho_{c}}
$$

where the current critical energy density is expressed in terms of current Hubble factor $H_{0}=H\left(\mathcal{T}_{0}\right)$ as:

$$
\rho_{c}=\frac{3 c^{2}}{8 \pi G} H_{0}^{2}
$$

From eq.(13) and with eqs.(8) and (14), we obtain:

$$
\Omega=\frac{2(d-1)}{3 d} \frac{\mathcal{T}_{0}^{-2}}{H_{0}^{2}}
$$


As like as $H_{0} \sim \mathcal{T}_{0}^{-1}$, we have finally

$$
\Omega=\frac{2(d-1)}{3 d}
$$

In our three-dimensional expanding Universe, it gives $\Omega=\frac{4}{9}$.

In the last, we have taken $\mathcal{T}_{0} \leq H_{0}{ }^{-1}$ following the usual computation. In General Relativity framework, it holds:

$$
\mathcal{T}_{0}=\frac{2}{3} H_{0}{ }^{-1}
$$

if the deceleration parameter $q_{0}=-\ddot{a}\left(t_{0}\right) \frac{a\left(t_{0}\right)}{\dot{a}\left(t_{0}\right)^{2}}>\frac{1}{2}$. For our model, the deceleration parameter is found:

$$
q_{0}=\frac{1-M}{M}
$$

that for standard matter dominated behaviour gives exactly $q_{0}=\frac{1}{2}$. For this and as well as observations give $q_{0} \sim 1$, we compute the value of $\Omega$ in this framework. From eq.(15) and eq.(17) we obtain:

$$
\Omega=\frac{2}{3} \frac{d-1}{d}\left(\frac{2}{3}\right)^{-2}
$$

which in the three dimensional case gives exactly:

$$
\Omega=1
$$

We have obtained that a metric spatially flat $k=0$ leds to a critical energy density $\Omega=1$. This result is so known in General Relativity, but here it has been extracted in a no General Relativity Framework, since low energy effective equations are not a priori equivalent to General Relativity equations. Looking from the point of view of the Brans-Dicke metric-dilaton coupling, General Relativity is obtained as the limit of the Brans-Dicke parameter $\omega \rightarrow \infty$, while the low energy effective string action (see eq.(11)) is obtained for $\omega=-1$.

Notice that $\Omega=1$ is obtained as a result of applying coherently General Relativity statements in the matter dominated stage, but the initial eq.(8) comes for the inflationary stage, described in the low energy effective string framework. Thus, a result from this string treatment is compatible with, and leads to similar predictions that, standard cosmology.

Since in anycase $\mathcal{T}_{0} \leq H_{0}{ }^{-1}$, eq.(8) can be seen as giving a lower limit for $\Omega$ :

$$
\Omega \geq \frac{2}{3} \frac{(d-1)}{d}
$$

This is the prediction for the current energy density from String Driven Cosmology and this is not in disagreement with the current observational limits for $\Omega$. 


\subsection{Discussion of the Model}

\subsubsection{String Driven Cosmology is Selfconsistent}

Matter dominated stages are not an usual result in string cosmology backgrounds. The standard time of matter dominated beginning $\mathcal{T}_{m} \sim 10^{12} s$ is supposed too late in order to account for string effects. But this is coherent with the fact that matter dominated stage is described selfconsistently by effective string treatments.

The General Relativity equations plus string sources supposes one of the weakest regimes for describing string-metric coupling. In this treatment, metric evolves following classical General Relativity equations and the string effect is accounted uniquely by considering them as the classical matter source ([2]). Backreaction happens since the equation of state for string behaviours have been obtained by studying their propagation in curved backgrounds. The result is selfconsistent because Einstein equations returns the correct curved background when aymptotic behaviours have been taken. Thus, it is a good point that the current stage appears selfconsistently as the asymptotic limit for large scale factor. Similarly, the radiation dominated stage is obtained from both effective treatments considered. This is coherent with having such stage previous to the current stage (where string effects must be not visible) and successive to a stage extracted under considerations of stronger string effects.

The intermediate behaviour between radiation and matter dominated stages is not known. Because this and current knowledge, this effective treatment is not able to describe suitably the radiation dominated-matter dominated transition. As it is said elesewhere [7], a sudden but continuous and smooth transition among both stages is not possible without an intermediate behaviour. Other comment must be dedicated to the inflation-radiation dominated transition. In the String Driven Model, this transition requires a brief temporaly exit of low energy effective regime for comeback within it at the beginning of radiation dominated. This could be understood as the conditions neccesary to modify the leading behaviour from unstable strings to dual strings. Further knowledge about the evolution of strings in curved backgrounds is necessary. It has been reported that multistring solutions (strings propagating in packets) are present in curved backgrounds and show different and evolving behaviours ( $13 \pi-15 \|)$. Research in this sense could aid to overcome the transition here considered, asymptotically rounded by low energy effective treatments. 


\subsubsection{String Driven Inflation realizes a Big-Bang}

During the inflationary stage, the scale factor suffers a great expansion, enough for solving the cosmological puzzles. The almost amount of expansion is reached around the exit time. In fact, the beginning of this stage is characterized for a very, very slow evolution for the scale factor and dilaton. This evolution increases speed in approaching the exit inflation, since it is approaching also the pole singularity in scale factor. From a phenomenological point of view, the inflationary scale factor describes a very little and very calm Universe emerging from the Planck scale. The evolution of this Universe is very slow at the beginning, but the string coupling with the metric and the string equation of state drive this evolution, leading to a each time more increasing and fast dynamics. In approaching the exit of inflation, the scale factor and the spacetime curvature increase. The metric "explodes" around the exit of inflation. The last part of this explosion is the transition to radiation dominated stage in a process that breaks transitorily the effective treatment of strings. This transition is supposed brief, the transition to standard cosmology happens at the beginning of radiation dominated stage.

At priori, this model seems privilege an unknown parameter $t_{I}$, playing the role usually assigned to singularity at $t=0$ in string cosmology. But this is not unnatural, since in order to reach an enough amount of inflation, this parameter is found to be very close to the standard radiation dominated beginning $t_{r}$ and so, to the beginning of standard cosmology. On the other hand, this value appears related to the GUT scale, which is consistent with the freezing of the dilaton evolution and change in the equation of state in our effective string treatment.

In this way, the Universe starts from a classical, weak coupling and small curvature regime. Driven by the strings, it evolves towards a quantum regime at strong coupling and curvature.

The argument above mentioned, where a brief transition exits and comebacks among stages in a low energy effective treatment, is not an exceptional feature in String Cosmology. Pre-Big Bang models [3] deal with two branches described in low energy effective treatments. Both branches are string duality related, but the former one runs on negative time values. The intermediate region of high curvature is supposed containing the singularity at $t=0$ and consequently, the Big Bang. Our String Driven Cosmology presents also this intermediate point of high curvature, but it is found around the inflation-radiation dominated transition, near but not on Planck scale. In our model, negative proper times are never considered and the instant $t=0$ remains before of the described inflationary stage (is not coherent include it in the effective description, since before Planck time a fully stringy regime

is expected whose effects can not be considered merely with the effective equations). There are not predicted singularities, neither at $t=0$ nor $t=t_{I}$, at the level of the 
minimal model here studied.

Another great difference with the "Pre-Big Bang scenario" is the predicted dynamics of universe. The Pre-Big Bang scenario includes a Dilaton Driven phase running on negative times. Not such feature is found in our Universe description. Although the curvature does not obey a monotonic regime, time runs always on positive values and the scale factor always expands in our String Driven Cosmology.

The Pre-Big Bang scenario assumes around $t \sim 0$ a "String Phase" with high almost constant curvature. Our minimal String Driven Cosmology does not assume such a phase, but a state of high curvature is approached (and reached) at the end of inflation stage. The low energy effective regime (L.E.E.) breaks down around the inflation exit, both due to increasing curvature (the scale factor approaches the pole singularity) and to the increasing dilaton. The exit of inflation and beginning of radiation dominated stage must be described with a more complete treatment for high curvature regimes.

From eq.(2), the growth reached by the dilaton field during the inflationary stage would not be so large, at least while the low energy effective treatment holds. The exact amount depends mainly on the initial inflationary conditions, the parameter $\phi_{I}$ being constrained by the effective equations (舟). In anycase, when the scale factor approaches its singularity and the $e$-folds number $f$ increases very quickly with time, the same is not true for the dilaton ratio. Comparatively, the dilaton ratio increases in a much slower way $\frac{\phi\left(t_{r}\right)}{\phi\left(t_{i}\right)} \sim f$ than the scale factor $\frac{a\left(t_{r}\right)}{a\left(t_{i}\right)} \sim e^{f}$. As a consequence, corrections due to the high curvature regime are needed much earlier than the corresponding to dilaton growth.

\section{Conclusions}

We have considered a minimal model for the evolution of the scale factor totally extracted from string cosmology. The earliest stages (an inflationary power type expansion and a radiation dominated stage) are obtained from the low energy effective equations, while the radiation dominated stage and matter dominated stage are selfconsistent solutions of the Einstein equations with a gas of strings as matter

sources. Such solutions suggest the low energy effective action is asymptotically valid at earliest stages, around and immediately after Planck time $t_{P} \sim 10^{-43} \mathrm{~s}$, when the spacetime and string dynamics would be strongly coupled. The radiation dominated stage is extracted from both treatments, coherently with being it an intermediate stage among the two regimes: inflation and matter dominated stage. On the other hand, since the stable string behaviour describes cold matter behaviour, current matter dominated stage can be extracted also in the string matter treat- 
ment. Notice that in string theory, the equation of state of the string matter is derived from the string dynamics itself and not given at hand from outside as in pure General Relativity.

No detail on the transitions dynamics can be extracted in this framework, too naive for accounting such effects. The inflation-radiation dominated transition implies a transitory breaking of the low energy effective regime. The radiation dominated-matter dominated stage can not be modelized in sudden, continuous and smooth way. Further study is required on the evolution of the equation of state for the gas of strings. The three string behaviours in curved backgrounds are present in this gas and each cosmological stage is selfconsistently driven by them. In this way, the transition between inflation and radiation dominated stage is related with the evolution from unstable to dual string behaviour, while the radiation dominatedmatter dominated transition could be driven by passing from the dual to stable behaviour.

Phenomenological information extracted from this String Driven model is compatible with observational Universe information. An amount of inflation, usually considered as enough for solving the cosmological puzzles, can be obtained in the inflationary stage. Energy ranges at exit of inflation are found coherents with GUT scales. The inflationary stage gives a value for the energy density-dilaton coupled term equivalent to the corresponding critical energy density, computed as in General Relativity. That means, we have $\left.\Omega\right|_{\text {inf }}=1$, whenever the end of inflationary stage be computed. Also, the contribution to current energy density is computed $\Omega \geq \frac{4}{9}$, and taking account the validity of General Relativity in the current matter dominated stage, we find this contribution be exactly $\Omega=1$.

Our main conclusion is to have proved that string cosmology, althought being effective, is able to produce a suitable minimal model for Universe evolution. It is possible to place each effective context in a time-energy scale range. Energy ranges are found and General Relativity conclusions are obtained too in a string theory context coherently. We have extracted the General Relativity statement about spatial curvature and energy density, at least for the spatially flat case $(k=$ $0 \rightarrow \Omega=1$ ) in a totally Non-Einstenian framework, like the low energy effective string action giving rise to the inflationary String Driven stage.

In their validity range, no need of extra stages is found. Only the interval around the transitions and the very beginning epoch, probably the Planck epoch, will require more accurate treatments that hitohere considered. Since the behaviours above extracted are asymptotic results, it is not possible to give the detail of the transitions among the different stages. The connection among asymptotic low energy effective regimes through a very brief stage (requiring a more complete description of string dynamics) enables us to suppose this stage containing the evolution in the equation of state from unstable strings domain to dual strings one. From the point of view of 
the scale factor evolution, this brief transition could be modelized as nearly instantaneous, provided curvature and scale factor expansion have attainted nearly their maximun values. Similar comment can be made in the radiation dominated-matter dominated transition. It must be driven by the subsequent evolution of the gas of strings from dual to unstable domain to string stable behaviour domain. Again, a brief intermediate stage could take place among both asymptotic behaviours. But this is an open question in the framework of string cosmology both for inflationradiation dominated as well as radiation dominated-matter dominated transition.

\section{ACKNOWLEDGMENTS}

M.P.I. wants to thank Marina Ramón Medrano and Héctor J. de Vega for very helpful discussions.

\section{References}

[1] A.A.Tseytlin in String Gravity and Physics at the Planck Energy Scale, Proceedings of the Erice Chalonge School 1992, ed. by N.Sánchez (World Scientific, Singapore 1993).

[2] H.J.De Vega and N.Sánchez, Phys.Rev.D 50 (1994) 7202.

[3] M.Gasperini and G.Veneziano, Astropart.Phys. 1 (1993) 317.

[4] String Theory in Curved Spacetimes, ed. by N.Sánchez (World Scientific, Singapore, 1998).

[5] String Gravity and Physics at the Planck Scale, Proceedings of the Erice Chalonge School, Sept. 1995, ed. by N.Sánchez and A.Zichichi, NATO ASI 476 C, Kluwer Ac. Publ. 1995.

[6] M.Gasperini and M.Giovannini, Phys.Rev.D 47 (1993) 1519.

[7] M.P.Infante and N.Sánchez, Linking a Minimal Theoretical Model of Scale Factor Evolution with Observational Universe Information (in preparation).

[8] M.Gasperini, N.Sánchez and G.Veneziano, Int. Jour. Mod. Phys. A 6 (1991) 3853.

[9] M.Gasperini, N.Sánchez and G.Veneziano, Nuc.Phys.B 364 (1991) 365.

[10] M.P.Infante and N.Sánchez, The Primordial Gravitational Wave Background in String Cosmology (accepted for publication in Phys.Rev.D).

[11] M.Gasperini and G.Veneziano, Mod.Phys.Lett. A 8 (1993) 3701. 
[12] N.Kaloper, A.Linde and R.Bousso, Phys.Rev.D 59 (1999) 4350.

[13] F.Combes, H.J.De Vega, A.V.Mikhailov and N.Sánchez, Phys.Rev.D 50 (1994) 2754.

[14] H.J.De Vega, A.Larsen and N.Sánchez, Nucl.Phys.B 427 (1994) 643.

[15] H.J.De Vega, A.Larsen and N.Sánchez, Phys.Rev.D 51 (1995) 6917.

[16] G.Veneziano, Phys.Lett.B 265 (1991) 287.

[17] H.J.De Vega and N.Sánchez, Phys.Lett.B 197 (1987) 320.

[18] N.Sánchez and G.Veneziano, Nuc.Phys.B 333 (1990) 253.

[19] M.Gasperini, Phys.Rev.D 56 (1997) 4815. 